\section{Prostate's PTEN prognosis}

\section{By Chris Cain, Staff Writer}

U.S. and European academics have separately identified new biochemical signatures that could improve prostate cancer diagnosis and monitoring. ${ }^{1,2}$ ProteoMediX AG holds rights to the European data and plans to develop a blood-based test that will complement PSA testing to increase diagnostic accuracy. Metamark Genetics Inc. has licensed the American team's results and plans to develop an objective histological test that will complement Gleason scoring to improve prognostic prediction.

The standard screening procedure to detect prostate cancer includes a blood test that measures levels of prostate-specific antigen (KLK3; PSA). ${ }^{3}$ Although PSA testing can detect prostate cancer with high sensitivity, the test has poor specificity. The reason for the high rate of false positives is that unrelated factors including prostate inflammation can increase

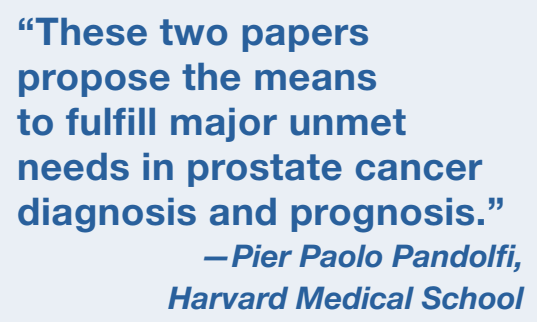

In collaboration with Ruedi Aebersold, professor and head of the Institute of Molecular Systems Biology at ETHZ, the group used mass spectrometry to identify and quantify glycosylated proteins in prostate tissue and serum collected from wild-type or Pten mutant mice. The authors limited their analysis to glycosylated proteins, which can be selected for during sample preparation, to maximize the approach's sensitivity.

After identifying 126 serum proteins that were expressed differently in Pten-deleted mouse prostate tissue compared with control tissue, the researchers set out to validate their findings in the clinic. They collected serum from 77 prostate cancer patients and 66 controls with benign prostatic hyperplasia $(\mathrm{BPH})$ and performed targeted mass spectrometry to measure the levels of candidate biomarkers in each patient group.

The ETHZ team also used computational modeling to find the sets of proteins that were most predictive of PTEN status, prostate cancer diagnosis or Gleason score. The approach identified a four-protein signature that could improve the accuracy of prostate cancer diagnosis when combined with PSA measurement.

Separately, each test distinguished prostate cancer from $\mathrm{BPH}$ with sensitivity and specificity of about $80 \%$ and $60 \%$, respectively. Combined, sensitivity and specificity increased to $85 \%$ and $79 \%$, respectively.

Results were published in the Proceedings of the National Academy of Sciences.

In a separate study, the Dana-Farber researchers sought to identify new prognostic PSA levels.

If serum PSA concentration exceeds a certain threshold, a patient may elect to undergo prostate biopsy, in which a tissue sample is extracted and examined microscopically for cellular abnormalities. A trained pathologist then determines the presence of cancerous cells and assigns a Gleason score that ranges from 2-10, with the upper number representing the worst prognosis.

Gleason scores are used to determine how aggressively to treat the cancer, with options that range from watchful waiting to radical prostatectomy. The score is a good way to measure progression but can vary depending on the pathologist who examines the sample.

To improve decision making, the academics at the Swiss Federal Institute of Technology Zurich (ETHZ) and the Dana-Farber Cancer Institute both used a mouse model of cancer induced by deleting Pten (Mmac1; Tep1) specifically in the prostate.

$P T E N$ is the second most frequently mutated tumor suppressor gene behind $p 53$, and genetic alterations of at least one PTEN allele have been reported in $70-80 \%$ of prostate cancers. ${ }^{4}$

"We didn't think that searching in a sea of diverse patients with different stages of cancer was the most rational strategy to identify new cancer markers," said Wilhelm Krek, a professor of cell biology at ETHZ who led one of the groups. "This mouse model is a highly reproducible, robust and controlled system that maximized our chance of identifying relevant prostate-specific biomarkers." markers for prostate cancer. Their first step was generating a doubleknockout mouse with exacerbated disease in which both Pten and mothers against decapentaplegic homolog 4 (Madh4; Smad4; Dpc4) were deleted in the prostate. SMAD4 is a DNA-binding transcriptional regulator thought to play a role in prostate cancer progression.

Whereas Pten-deleted mice developed localized lesions in the prostate that were only minimally metastatic, the double knockouts developed aggressive metastatic disease. Subsequent analyses showed that the aggressive disease partly stemmed from upregulation of cyclin D1 (Ccnd1; Bcl1) and osteopontin (Opn; Spp1), a pair of targets downstream from Smad4.

Those two targets increased tumor growth and metastasis when expressed in prostate tumor cells that were implanted into mice.

The prognostic value of all four proteins was validated by immunohistochemical staining of tissue samples from 405 tumor specimens from men diagnosed with prostate cancer who underwent radical prostatectomy. Staining for the four proteins predicted clinical outcome significantly better than Gleason scoring, and the combination of the two measures further increased prognostic accuracy compared with either alone.

Results were published in Nature.

Study leader Ronald DePinho, professor of medicine and genetics at Harvard Medical School and director of the Belfer Institute for 
Applied Cancer Science at Dana-Farber, told SciBX the goal was to "identify genes that were functional determinants of cancer progression because we thought they would be the most accurate predictors of prognosis."

"These two papers propose the means to fulfill major unmet needs in prostate cancer diagnosis and prognosis," said Pier Paolo Pandolfi, professor of medicine and pathology at Harvard Medical School and director of research at the Cancer Center at Beth Israel Deaconess Medical Center who has pioneered the development of Pten-deficient mouse models.

Pandolfi noted that even prostate cancers without PTEN mutation frequently have decreased expression of PTEN, suggesting that these gene signatures could be applicable to many patients regardless of PTEN mutational status.

Pandolfi did say that large, multicenter prospective trials are needed to validate the findings.

"One advantage of prostate cancer is that there is a great lag between diagnosis and eventual outcome, so it provides an opportunity to follow patients' progress and collect samples over time, making it easier to validate and compare tests with respect to other, more aggressive cancers," he said.

\section{Diagnosing commercial development}

ProteoMediX was founded in 2010 as a spinoff of ETHZ to develop a test based on the candidate glycoprotein signatures identified in the PNAS publication. The company is developing ELISA-based assays for prostate cancer based on the findings and hopes to begin pivotal clinical trials in two to three years.

"Our big advantage is we already know the signature we want to look for, and we now need to develop the assays," CEO and cofounder Ralph Schiess told SciBX. "There is a huge demand for improved tests for prostate cancer diagnosis, and while mass spectrometry is a powerful discovery tool, it would not have the throughput necessary to handle that demand."

"While the company is developing the commercial aspect, at ETH Zurich we will continue to use mass spectrometry to examine this signature in larger patient cohorts, to look for new and improved signatures and to test whether we can predict PTEN mutation status in other cancer types," said Krek, also a cofounder of ProteoMediX.

Metamark was founded in 2007 to develop prognostic tests for cancer based on the work of DePinho and Lynda Chin, a professor of dermatology at Harvard Medical School and scientific director of the Belfer Institute who also was an author of the Nature paper.
CSO Peter Blume-Jensen told SciBX that the company "is developing tests that use quantitative immunofluorescence to accurately measure protein levels in tissue samples, and we intend to automate the assays as much as possible. Gleason scoring is somewhat subjective and can vary from doctor to doctor, and we want to try to remove that element of uncertainly in favor of an evidence-based, quantitative test."

He said the Nature paper "lays a strong biochemical foundation to build a prognostic test that can complement or replace Gleason scoring, to help determine the risk of prostate cancer progression and recurrence before treatment decisions are made."

The company plans to finalize its choice of markers by the end of 2011 and to launch a validation study in 2Q12.

"The company goes beyond looking at correlative prognostic biomarkers-it is trying to look at functionally significant drivers of cancer aggression," said Chin. "If you look at validated diagnostic and prognostic biomarkers in clinical use, every single one is functionally important. They are not just correlative; they also play a role in disease."

Cain, C. SciBX 4(9); doi:10.1038/scibx.2011.241

Published online March 3, 2011

\section{REFERENCES}

1. Ding, Z. et al. Nature; published online Feb. 2, 2011; doi:10.1038/nature09677

Contact: Ronald A. DePinho, Dana-Farber Cancer Institute, Boston, Mass.

e-mail: ron_depinho@dfci.harvard.edu

Contact: Lynda Chin, same affiliation as above

e-mail: lynda_chin@dfci.harvard.edu

2. Cima, I. et al. Proc. Natl. Acad. Sci. USA; published online Feb. 7, 2011; doi:10.1073/pnas.1013699108

Contact: Wilhelm Krek, Swiss Federal Institute of Technology Zurich (ETHZ), Zurich, Switzerland

e-mail: wilhelm.krek@cell.biol.ethz.ch

Contact: Ruedi Aebersold, same affiliation as above

e-mail: aebersold@imsb.biol.ethz.ch

Contact: Holger Moch, University Hospital Zurich, Zurich, Switzerland e-mail: holger.moch@usz.ch

Contact: Silke Gillessen, Cantonal Hospital St. Gallen, St. Gallen,

Switzerland

e-mail: silke.gillessen@kssg.ch

3. Wolf, A.M.D. et al. CA Cancer J. Clin. 60, 70-98 (2010)

4. Gray, I.C. et al. Br. J. Cancer 78, 1296-1300 (1998)

\section{COMPANIES AND INSTITUTIONS MENTIONED}

Cancer Center at Beth Israel Deaconess Medical Center,

Boston, Mass.

Dana-Farber Cancer Institute, Boston, Mass.

Harvard Medical School, Boston, Mass.

Metamark Genetics Inc., Cambridge, Mass.

ProteoMediX AG, Zurich, Switzerland

Swiss Federal Institute of Technology Zurich, Zurich, Switzerland 